\title{
ANN Modeling of Monthly and Weekly Behaviour of the Runoff of Kali River Catchment
}

\author{
Eswara Reddy Orekanti \\ Associate Professor and Head, Department of Civil Engineering, Sree Vidyanikethan Engineering College, \\ Sree Sainath Nagar, A. Rangampet - 517 102, Tirupati, A.P., INDIA; orekantieswar@gmail.com
}

\begin{abstract}
Model is a system, by whose operation; the characteristics of other similar systems can be ascertained. Experimental observation made on a model bear a definite relationship with prototype. So, the model analysis or modeling is actually an experimental method of finding solution of complex flow problems like surface water modeling, sub-surface water modeling etc. Many flow situations are not amenable to theoretical analysis. Modeling is a valuable means of obtaining better understanding of particular situation. Inspired by the functioning of the brain and biological nervous system, Artificial Neural Networks (ANNs) has been applied to various hydrological problems in last two decades. In this study, two ANN models using feed forward - back propagation network are developed to correlate a relationship between rainfall and runoff on monthly and weekly basis for Kali river catchment up to Supa dam in Uttara Kannada District of Karnataka State, India. The developed two models are compared and evaluated using standard statistical parameters to know strength and weaknesses. This performance can be further refined by incorporating more input parameters of catchment properties like soil moisture index; land use and land cover details etc.
\end{abstract}

Keywords: Rainfall-Runoff Modeling, Artificial Neural Networks, Mean Average Error (MAE), Root Mean Square Error (RMSE), Relative Mean Average Error (RMAE), Catchment.

\section{Introduction}

The rainfall-runoff relationships are among the most complex phenomena to comprehend to due to tremendous spatial and temporal variability of watershed characteristics, precipitation patterns as well as number of variables involved in modeling the physical processes. For many years, hydrologists have attempted to understand transformation of rainfall to runoff in order to forecast stream flow for water supply, flood control, irrigation, drainage, water quality, power generation, recreation and wildlife propagation. The transformation of precipitation into runoff involves many highly complex components such as interception, depression storage, infiltration, overland flow, interflow, percolation, evaporation and transpiration. Many models have been developed to simulate this process. These models can be categorised as empirical, conceptual and physical. Each of these models has its own advantages and limitations. Many situations in practice demand use of simple tools such as linear theoretic models or empirical models or black box models. However, these simple models normally fail to represent non-linear dynamics which are inherent in the process of rainfall-runoff transformation. The adoption of ANN technique for rainfall-runoff modeling has added a new dimension to system theoretic modeling approach and it has been applied in recent years as a successful tool to solve various problems concerned with hydrology and water resources engineering $\{[1],[2]\}$.

Artificial Neural Networks have been in existence since 1940's, but since current algorithms have overcome the limitations of these early networks in the great interest of practical applications of ANNs has arisen in recent decades (Wasserman 1989) [3]. Various ANN algorithms have an object to map a set of inputs to a set of outputs. An ANN is described as an information processing system that is composed of many nonlinear and densely interconnected processing elements or neurons. ANNs have been proven to provide better solutions when applied to (i) complex system that may be poorly described or understood (ii) problems that deal with noise or involve pattern recognition, diagnosis, abstraction and generalisation and (iii) situations where the input is incomplete or ambiguous by nature. It has been reported that an ANN has the ability to extract patterns in phenomenon and over come difficulties due to the selection of a model form such as linear, power or polynomial. An ANN algorithm is capable of modeling rainfall-runoff relationship due to ability to generalise patterns in noisy and ambiguous input data and synthesize a complex model without prior knowledge or probability distributions. The ANN model is calculated using automatic calibration techniques. Thus, an ANN model eliminates subjectivity and lengthy calibration procedure.

French et al (1992) [4] developed a three layer feed forward network to forecast rainfall intensity in space and time and compared the results with two other methods for short term forecasting. Hsu et al (1995) [5] have found the multilayer feed forward network to be the best input-output function approximation. They proposed a linear least square simplex algorithm to train a three-layer feed forward network and demonstrated the potential of such models for simulating the hydrologic behaviour of a watershed. They also showed that the 
ANN model approach provides a better representation of the rainfall-runoff relationship of a medium sized basin than the ARMAX model or Sacramento soil moisture model. Raman and Sunil Kumar (1995) [6] investigated the use of ANNs for synthetic inflow generation and compared their performance with multivariate time series model. Raman and Chandramouli (1996) [7] derived reservoir operating policies for a dam in India.

Wilby et al (1998) [8] used ANNs for river flow forecasting and highlighted the ability of ANNs to cope with missing data and learn from the event currently being forecast in real time. They also emphasized the need for thorough investigation of relationship between the trained period length and hydrological realism of the ANN forecast. Toker and Johnson (1999) [9] employed ANN methodology to forecast daily runoff as a function of daily precipitation, temperature, and snowmelt for the Little Patuxent River watershed in Maryland. The sensitivity of the prediction accuracy to the content and length of training data was investigated. The ANN rainfall-runoff model compared favorably with results obtained using existing techniques including statistical regression and a simple conceptual model. Sajikumar and Thandaveswara (1999) [10] conclude that an ANN was the most efficient of black-box models test for calibration periods as short as 6 years. Toker and Markus (2000) [11] applied ANN and conceptual models like Watbal model, SAC-SMA model and SCRC model for three catchments and demonstrated that ANNs can accurately model nonlinear relationship between hydrologic inputs and output. Jain and Chalisgaonkar (2000) [12] applied Three-layer feed forward ANNs to model riverrating curves in order to establish a stage-discharge relation as part of the processing of streamflow data. Rajurkar et al (2004) [13] used ANNs along with an auxiliary model to predict daily rainfall for seven catchments taken worldwide.

In this study, an ANN algorithm was used to model the weekly and monthly rainfall-runoff relationship for Supa dam catchment of Kali River (Fig.1) in Uttara Kannada District of Karnataka State, India. The networks were trained and tested using data that represent different rainfall patterns. The sensitivity of the network performance to the content and length of calibration data was examined using various training data sets.

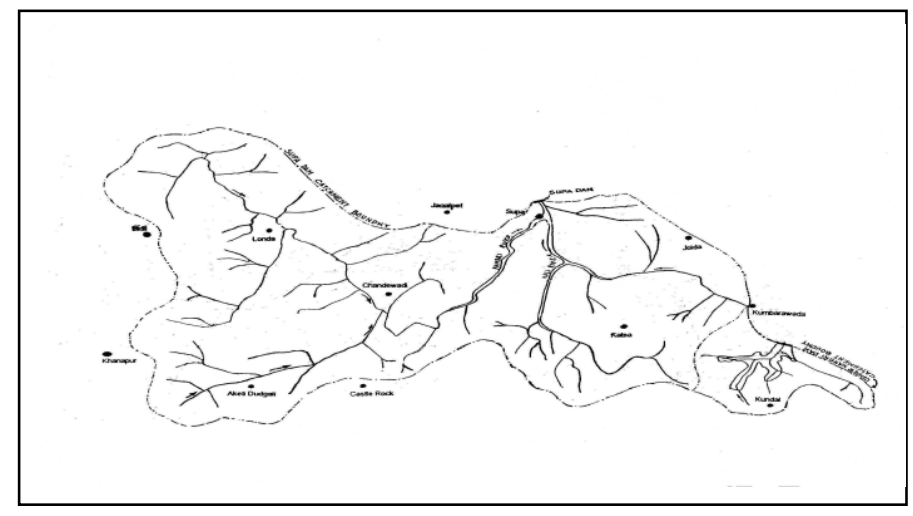

Fig. 1 Supa dam catchment area of Kali River

\section{Review of ANNs}

An ANN is a network of parallel, distributed information processing systems that relate an input vector to an output vector. It consists of a number of information processing elements called neurons or nodes, which are grouped in layers. The input layer processing elements receive the input vector and transmit the value to the next layer of processing elements across connections where this process is continued. This type of network, where data flow one way (forward way), is known as a feed forward network. The basic structure of a network usually consists of three layers - the input layer, where the data are introduced to the network; the hidden layer (s), where data are processed and the output layer, where the results for given inputs are produced [14].

The most distinctive characteristic of an ANN is its ability to learn from examples. Learning or training is defined as self adjustment of the network weights in order to approximate the target output (observed or measured output) based on certain algorithm. Learning in ANNs consists of three elements - weights between neurons that define the relative importance of the inputs, a transfer function (generally a sigmoid function) that controls the generation of the output from a neuron, and learning laws that describe how the adjustments of the weights are made during training. During learning, a neuron receives inputs from the input or previous layer, weights each input with a pre-assigned value and combines these weighted inputs. The combination of the weighted inputs is represented as

$$
\text { net }_{\mathrm{j}}=\sum w_{i j} x_{i}
$$


Where net $t_{\mathrm{j}}=$ summation of weighted input for $j$ th neuron; $\mathrm{w}_{\mathrm{ij}}=$ weight from $i^{\text {th }}$ neuron in the previous layer to the $j^{\text {th }}$ neuron in the current layer; and $\mathrm{x}_{\mathrm{i}}=$ input from $i^{\text {th }}$ to $j^{\text {th }}$ neuron. The net $t_{\mathrm{j}}$ is either compared to a threshold or passed through a transfer function to determine the level of activation. The activation of a neuron is strong enough; it produces an output that is sent as an input to the other neurons in the successive layer. In this study, the training of ANNs is accomplished by a back-propagation algorithm. The back-propagation is the most commonly used supervised training algorithm in the multilayer-feed forward networks. Here information is processed in the forward direction from the input layer to the hidden layer (s) and then to the output layer. The objective of a back-propagation network is to find the weights that approximate target values of input with a selected accuracy. The least mean square error method along with generalised delta rule is used to optimise network weights in back-propagation networks. The gradient descent method, along with chain rule of the derivative, is employed to modify the network weights.

\section{Model Development}

Measurements of precipitation, air temperature and stream discharge can be obtained easily, therefore a model that uses available real time data would be more easily applied in the operational forecast systems. Variables - precipitation, air temperature were selected to describe the physical phenomenon of rainfall-runoff process, in order to forecast stream discharge. In this study, the eight year rainfall data pertains to south-west monsoon months (June to October) was selected for training and testing. Recent data were used whenever possible since they reflect the current land-use conditions in the watershed. The most current data were used in the test set to illustrate the capability of model in predicting future occurrences of runoff, without directly including the land-use characteristics of watershed. Weekly and monthly rainfall-runoff processes were modeled using networks with a hidden layer. The ANN results were transformed back to the original domain and the final model structures were then used to calculate various statistical performance evaluation criteria using both training and testing data sets. Three different standard statistical performance evaluation criteria were used to evaluate model relative strengths and weaknesses of the models developed in the present study. These are mean average error (MAE), root mean square error (RMSE) and relative mean average error (RMAE) (Yen-Ming Chiang et al 2004) [15].

\section{Results and Discussions}

Table 1 summarises the results obtained from the ANN models developed using the inputs and provide a comparison of results. The results show that both models produce good forecasting for stream flow in both training and testing phases. In training phase monthly model performed slightly better than weekly model. But overall manner monthly model predicted runoff well. This indicates that the monthly model performance is relatively stable than weekly model. The RMSE statistic is a measure of residual variance and is indicative of the model's ability to predict high flows. Considering the magnitude of the peak flow during the period of study $\left(1268.02 \mathrm{~m}^{3} / \mathrm{s}\right.$ and $\left.620.6 \mathrm{~m}^{3} / \mathrm{s}\right)$ was predicted quite accurately $\left(1249.98 \mathrm{~m}^{3} / \mathrm{s}\right.$ and $\left.620.5 \mathrm{~m}^{3} / \mathrm{s}\right)$ can be evidenced by low RMSE values in Table 1. However it is worth noting that both the models have lower efficiency during testing suggesting large amount of unexplained variance. This lower efficiency indicates that model prediction away from the mean would not be accurate.

Table 1: Values of cross validation and efficiency for developed models

\begin{tabular}{|c|c|c|c|c|c|c|c|c|c|c|}
\hline \multirow{2}{*}{$\begin{array}{l}\text { ANN } \\
\text { Model }\end{array}$} & \multirow{2}{*}{$\begin{array}{l}\text { Archi- } \\
\text { tecture }\end{array}$} & \multirow{2}{*}{$\begin{array}{l}\text { No. of } \\
\text { Cycles }\end{array}$} & \multicolumn{2}{|c|}{ MAE } & \multicolumn{2}{|c|}{ RMSE } & \multicolumn{2}{|c|}{ RMAE } & \multicolumn{2}{|c|}{ Correlation Coefficient } \\
\hline & & & Training & Testing & Training & Testing & Training & Testing & Training & Testing \\
\hline Monthly & $24-3-1$ & 150000 & 0.083 & 0.094 & 0.5252 & 1.96 & 0.01 & 0.503 & 0.97 & 0.944 \\
\hline Weekly & $24-3-1$ & 150000 & 0.031 & 0.068 & 0.5273 & 1.89 & 0.01 & 0.097 & 0.943 & 0.8616 \\
\hline
\end{tabular}

Fig.2 and Fig.3 depicts the scatter plot of weekly and monthly runoff. The scatter plots show the reasonable and consistent performance in the two phases (training and testing). It is also observed that models are able to predict runoff at lower and higher levels reasonably well, but at middle level shows some deviation. This may be due to non-availability of wide range of precipitation patterns for ANN input during training phase. 


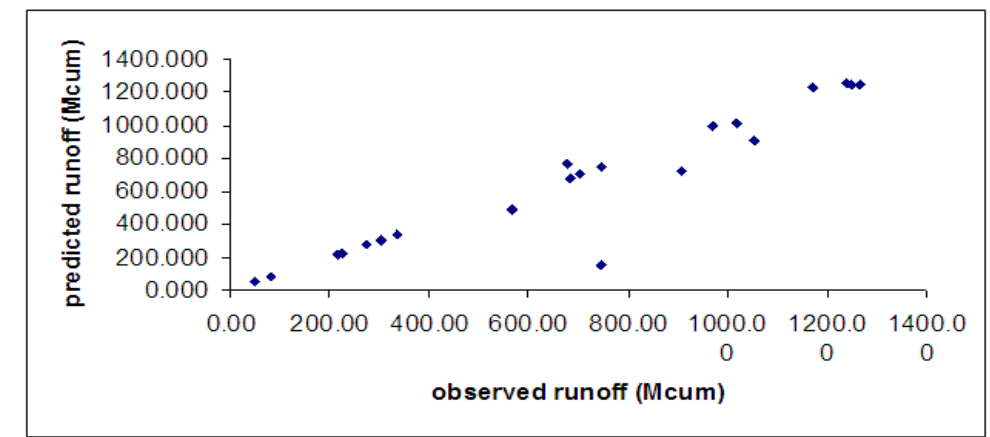

Fig. 2 Scatter plot for monthly runoff (training $\&$ testing period)

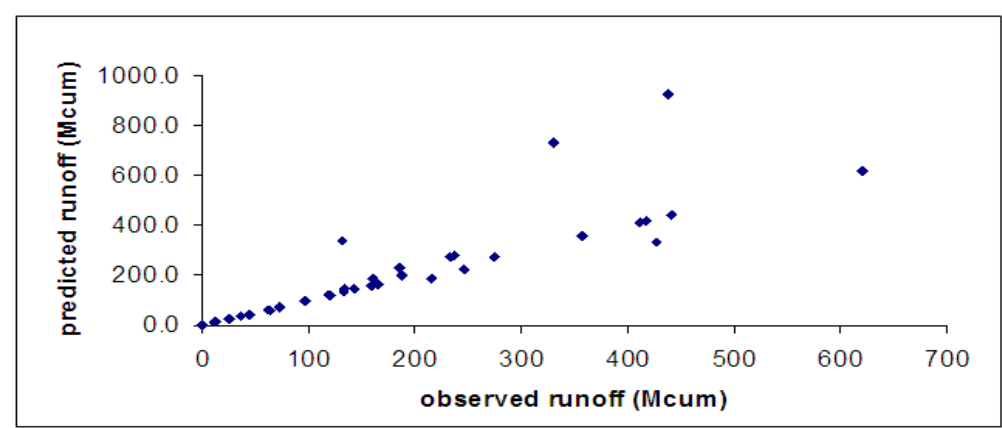

Fig. 3 Scatter plot for weekly runoff (training \& testing period)

\section{Conclusions}

Artificial Neural Networks is the new tool emerged out for watershed modeling owning to its advantage over other conceptual models. In present study multilayered (feed forward back propagation technique) ANN is used for rainfall - runoff modeling of Kali River Up to Supa Dam. Out of the 2 models developed monthly model performing slightly better with a success rate of $95.7 \%$ to that of weekly model with $91.67 \%$. This slight deviation may be attributed to bias values in testing phase of model. Further the overall efficiency of the 2 models can be improved by increasing the input data like soil moisture index, land cover \& land use details of the catchment. In this regard other multilayered tools like dynamic feed forward, real time recurrent learning algorithm may be helpful.

\section{References}

[1] ASCE Task Committee on Application of the Artificial Neural Networks in Hydrology (2000a), Artificial neural networks in hydrology I: preliminary concepts, Jnl. of Hydrologic Engng., ASCE, 5(2), 2000, 115-123.

[2] ASCE Task Committee on Application of the Artificial Neural Networks in Hydrology (2000b), Artificial neural networks in hydrology II: hydrologic applications, Jnl. of Hydrologic Engng., ASCE, 5(2), 2000, 124-137.

[3] Wasserman, P. D., Neural computing theory and practice (Van Nostran Reinhold, New York, 1989).

[4] French, M. N., Krajewski, W. F. and Cuykendall, R. R., Rainfall forecasting in space and time using neural networks, Jnl. of Hydrology, 137, 1992, 1-31.

[5] Hsu, K. L., Gupta, H. V. and Sorooshian, S., Artificial neural network modeling of the rainfall runoff process, Water Resour. Res., 31(10), 1995, 2517-2530.

[6] Raman, H. and Sunil Kumar, N., Multivariate modeling of water resources time series using artificial neural networks, Hydrol. Sc. J., 40(2), 1995, 145-163.

[7] Raman, H. and Chandramouli, V., Deriving a general operating policy for reservoir using neural network, Journal of Water Resources Planning and Management, ASCE, 122(5), 1996, 342-347.

[8] Wilby, R. L., Abrahart, R. J and Dawson, C. W., Detection of conceptual model rainfall-runoff processes inside an artificial neural network, Hydrol. Sc. J., 48(2), 2003, 163-181.

[9] Tokar, A. S. and Johnson, P. A., Rainfall-runoff modeling using artificial neural networks, Jnl. of Hydrologic Engng., ASCE, 4(3), 1999, 232-239.

[10] Sajikumar, N. and Thandaveswara, B. S., A non-linear rainfall-runoff model using an artificial neural network, Jnl. of Hydrology, 216, 1999, 32-55.

[11] Tokar, A. S. and Markus, M., Precipitation-runoff modeling using artificial neural networks and conceptual models, Jnl. of Hydrologic Engng., ASCE, 5(2), 2000, 156-161.

[12] Jain, S. K. and Chalisgaonkar, D., Setting up stage-discharge relations using ANN, Jnl. of Hydrologic Engng., ASCE, 5(4), 2000, 428-433.

[13] Rajurkar, M. P., Kothyari, U. C. and Chaube, U.C., Modeling of the daily rainfall - runoff relationship with artificial neural network, Jnl. of Hydrology, 285, 2004, 96 - 113.

[14] Haykin S., Neural networks: a comprehensive foundation (Mac-Millan College Publishing Co., New York, 1994).

[15] Yen - Ming Chiang, Li - Chiu Chang and Fi - John Chang, Comparison of static-feed forward and dynamic-feedback neural networks for rainfall-runoff modeling, Jnl. of Hydrology, 290, 2004, 297 - 311. 